\title{
Effects of Different Annealing Gases on Pentacene OTFT With HfLaO Gate Dielectric
}

\author{
L. F. Deng, P. T. Lai, Member, IEEE, W. B. Chen, J. P. Xu, Y. R. Liu, H. W. Choi, and C. M. Che
}

\begin{abstract}
Pentacene organic thin-film transistors (OTFTs) with $\mathrm{HfLaO}$ high- $\kappa$ gate dielectric were fabricated. The dielectric was prepared by a sputtering method and then annealed in $\mathrm{N}_{2}, \mathrm{NH}_{3}$, $\mathrm{O}_{2}$, or $\mathrm{NO}$ at $400{ }^{\circ} \mathrm{C}$. The carrier mobility of the $\mathrm{NH}_{3}$-annealed OTFT could reach $0.59 \mathrm{~cm}^{2} / \mathrm{V} \cdot \mathrm{s}$, which is higher than those of the other three devices. Moreover, the $\mathrm{NH}_{3}$-annealed OTFT obtained the smallest subthreshold swing of $0.26 \mathrm{~V} / \mathrm{dec}$ among them. Furthermore, $1 / f$ noise measurement indicated that the $\mathrm{NH}_{3}$-annealed OTFT achieved the smallest $1 / f$ noise. All these should be attributed to the improved interface between the gate dielectric and the organic semiconductor associated with the passivation effects of the $\mathrm{NH}_{3}$ annealing on the dielectric surface.
\end{abstract}

Index Terms-Dielectric, high- $\kappa$, organic thin-film transistor (OTFT), $1 / f$ noise.

\section{INTRODUCTION}

$\mathbf{O}$ RGANIC thin-film transistor (TFT) (OTFT) has attracted wide attention in the academic and industrial fields [1], [2]. In comparison with its inorganic counterparts, OTFT has its own advantages. It can be fabricated on a flexible plastic substrate and can be realized in a large area at a low temperature. Moreover, since lithography is unnecessary, the cost can be greatly reduced. OTFT has potential to be applied to large-area flexible displays [3], RF-ID cards [4], electronic papers, and so on. Pentacene OTFT based on a $\mathrm{SiO}_{2}$ dielectric has achieved a performance that is comparable to that of an amorphous-silicon TFT from the view of carrier mobility [5]. However, the operating voltage is usually too high $(>15 \mathrm{~V})$ because of the low dielectric constant $(\kappa)$ of $\mathrm{SiO}_{2}$. The high operating voltage leads to large power consumption, which is

Manuscript received September 16, 2010; revised October 2, 2010; accepted October 3, 2010. Date of publication November 18, 2010; date of current version December 27, 2010. This work was supported in part by the RGC of Hong Kong under Project HKU 7133/07E, by the URC for Seed Fund for Strategic Research Theme of HKU on Molecular Materials, and by the University Development Fund (Nanotechnology Research Institute) of The University of Hong Kong under Grant 00600009 . The review of this letter was arranged by Editor C.-P. Chang.

L. F. Deng, P. T. Lai, and H. W. Choi are with the Department of Electrical and Electronic Engineering, The University of Hong Kong, Pokfulam, Hong Kong (e-mail: lfdeng@eee.hku.hk; laip@eee.hku.hk; hwchoi@eee.hku.hk).

W. B. Chen and C. M. Che are with the Department of Chemistry, The University of Hong Kong, Pokfulam, Hong Kong (e-mail: wbchen@eee.hku.hk; cmche@hkucc.hku.hk).

J. P. Xu is with the Department of Electronic Science and Technology, Huazhong University of Science and Technology, Wuhan 430074, China (e-mail: jpxu@mail.hust.edu.cn).

Y. R. Liu is with the School of Electronic and Information Engineering, South China University of Technology, Guangzhou 510641, China (e-mail: yrliu@eee.hku.hk).

Color versions of one or more of the figures in this letter are available online at http://ieeexplore.ieee.org.

Digital Object Identifier 10.1109/LED.2010.2087314 particularly unacceptable for portable equipment. To solve this problem, an insulator with high $\kappa$ value was prepared for the gate dielectric of OTFTs [6]-[8]. HfLaO has been reported to have less Fermi-energy pinning than $\mathrm{HfO}_{2}$ in MOSFETs [9], [10], and the $\kappa$ value of the former could be over 20. Furthermore, the subthreshold swing (SS) for OTFTs based on HfLaO was reported to be superior to that of polysilicon TFTs [11]. The carrier transport in the OTFTs is known to be decisively determined by the interface between the gate dielectric and the organic semiconductor. Therefore, in this letter, the effects of annealing the $\mathrm{HfLaO}$ gate dielectric in $\mathrm{N}_{2}, \mathrm{NH}_{3}, \mathrm{O}_{2}$, or $\mathrm{NO}$ on pentacene OTFTs are studied, and it is found that the OTFT treated in $\mathrm{NH}_{3}$ displays the best performance in terms of carrier mobility, SS, and $1 / f$ noise characteristics.

\section{EXPERIMENTAL Details}

A silicon substrate (n-type, $\langle 100\rangle$, resistivity of $0.5-0.7 \Omega$. $\mathrm{cm}$ ) was cleaned according to the standard RCA method. Next, the wafer was dipped into 5\% hydrofluoric acid to remove the native oxide. In the following, the wafer was washed by deionized water and dried in the common nitrogen stream. Subsequently, the wafer was inserted in the chamber of a sputterer (Denton Vacuum LLC Discovery 635). After the vacuum in the chamber was reduced to below $2 \times 10^{-6}$ torr, a $\mathrm{HfLaO}$ film was prepared by RF sputtering $(30 \mathrm{~W})$ of a HfLa target $(\mathrm{Hf}: \mathrm{La}=$ $6: 4$ in molar ratio) in argon $(24 \mathrm{sccm})$ and oxygen $(3 \mathrm{sccm})$ to form the gate dielectric. Then, the samples were treated by annealing to improve the surface and bulk characteristics of the $\mathrm{HfLaO}$ film. The samples were annealed in $\mathrm{N}_{2}, \mathrm{NH}_{3}, \mathrm{O}_{2}$, or $\mathrm{NO}$, respectively, at a flow rate of $1000 \mathrm{~mL} / \mathrm{min}$ and $400{ }^{\circ} \mathrm{C}$ for $10 \mathrm{~min}$. Next, the back oxide of the wafer was removed with 20\% hydrofluoric acid. Then, pentacene (purchased from Sigma-Aldrich) was evaporated on the dielectric by an evaporator (Edwards Auto 306). During the evaporation process, the substrate was kept at room temperature. The thickness of the pentacene film was $30 \mathrm{~nm}$ which was measured by a quartzcrystal oscillator, and the deposition rate was $1.2 \mathrm{~nm} / \mathrm{min}$. Finally, drain and source electrodes were formed by gold evaporation through a shadow mask at a vacuum $8 \times 10^{-6}$ torr. The channel width and channel length of the shadow mask were 200 and $30 \mu \mathrm{m}$, respectively.

The $I-V$ characteristics and noise characteristics of the OTFTs were measured by an HP 4145B semiconductor parameter analyzer, a Berkeley Technology Associates 9603 fieldeffect transistor (FET) noise analyzer, and an HP 35665A dynamic signal analyzer. The $C-V$ characteristics of the $\mathrm{Au}$-pentacene-HfLaO-Si structure were measured by an HP 


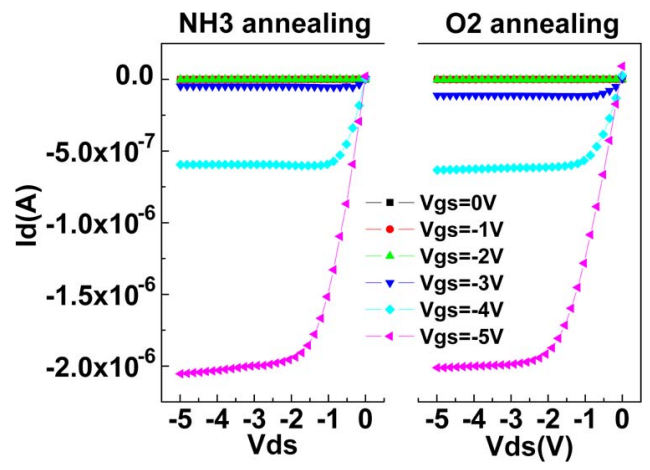

Fig. 1. Comparison of the output characteristics of the $\mathrm{NH}_{3}$ - and $\mathrm{O}_{2}$-annealed OTFTs.

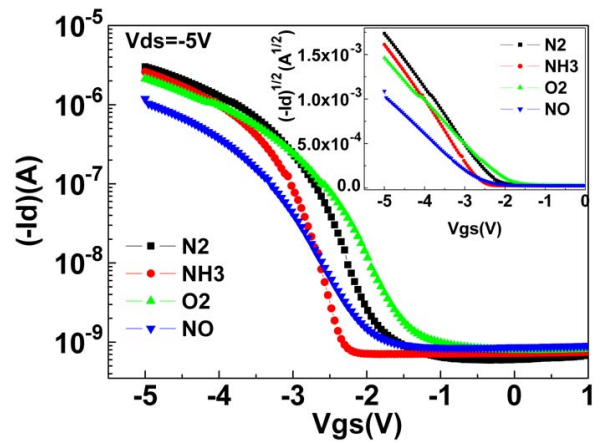

Fig. 2. Comparison of the transfer characteristics of the OTFTs. (Inset) $\left(-I_{d}\right)^{1 / 2}-V_{\text {gs }}$ curve.

4284A precision LCR meter. All the electrical measurements were completed in a shielded probe station under ambient atmosphere. The thickness of the dielectric films was derived by a WVASE32 ellipsometer made by the J. A. Woollam Company, Inc.

\section{RESUlts AND Discussion}

Fig. 1 shows the output characteristics of the OTFTs annealed in $\mathrm{NH}_{3}$ or $\mathrm{O}_{2}$. From these graphs, both OTFTs show good field-effect characteristics and could work well with a low supply voltage of $5 \mathrm{~V}$.

Fig. 2 shows the comparison of the transfer characteristics of the OTFTs with dielectric treated in $\mathrm{N}_{2}, \mathrm{NH}_{3}, \mathrm{O}_{2}$, or $\mathrm{NO}$, respectively. The carrier mobility $\mu$ and threshold voltage $V_{\text {th }}$ can be extracted from $\left(-I_{d}\right)^{1 / 2}$ versus $V_{\mathrm{gs}}$ in the saturation region for FETs [12]. The carrier mobility is given in (1), and the threshold voltage can be derived from the interception on the $x$-axis by extrapolating $\left(-I_{d}\right)^{1 / 2}$ versus $V_{\mathrm{gs}}$ to the $x$-axis

$$
\mu=\frac{2 L}{W C_{\mathrm{ox}}}\left(\frac{\partial \sqrt{-I_{d}}}{\partial V_{\mathrm{gs}}}\right)^{2}
$$

The carrier mobility of the $\mathrm{NH}_{3}$-annealed OTFT is $0.59 \mathrm{~cm}^{2} / \mathrm{V} \cdot \mathrm{s}$, which is higher than $0.49 \mathrm{~cm}^{2} / \mathrm{V} \cdot \mathrm{s}$ of the $\mathrm{N}_{2}$-annealed OTFT, $0.34 \mathrm{~cm}^{2} / \mathrm{V} \cdot \mathrm{s}$ of the $\mathrm{O}_{2}$-annealed OTFT, and $0.22 \mathrm{~cm}^{2} / \mathrm{V} \cdot \mathrm{s}$ of the NO-annealed OTFT. The switching characteristics of the OTFTs can be evaluated by their SS. The $\mathrm{NH}_{3}$-annealed OTFT obtains the smallest SS among the devices, thus the best switching characteristics.

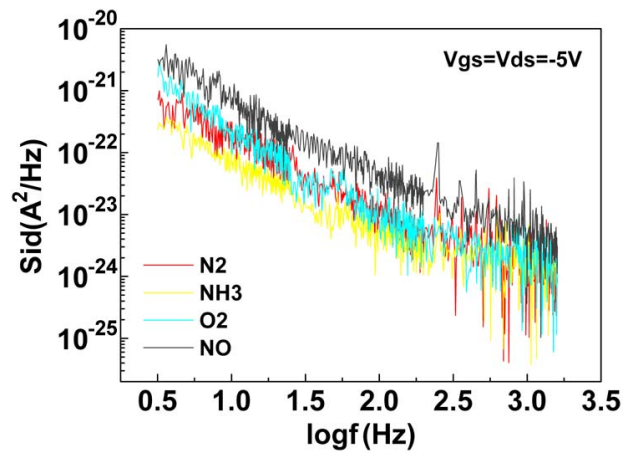

Fig. 3. Comparison of the $1 / f$ noise spectrum of the OTFTs annealed in $\mathrm{N}_{2}$, $\mathrm{NH}_{3}, \mathrm{O}_{2}$, or $\mathrm{NO}$.

TABLE I

DEVICE PARAMETERS OF THE OTFTS AND CAPACITORS

\begin{tabular}{lllll}
\hline \hline & $\mathrm{N}_{2}$ & $\mathrm{NH}_{3}$ & \multicolumn{1}{c}{$\mathrm{O}_{2}$} & $\mathrm{NO}$ \\
\hline mobility $\left(\mathrm{cm}^{2} / \mathrm{Vs}\right)$ & 0.49 & 0.59 & 0.34 & 0.22 \\
threshold voltage $(\mathrm{V})$ & -2.27 & -2.59 & -2.01 & -2.56 \\
$\mathrm{SS}(\mathrm{V} /$ dec $)$ & 0.34 & 0.26 & 0.45 & 0.60 \\
on/off current ratio $\left(10^{3}\right)$ & 5.1 & 3.7 & 2.7 & 1.4 \\
$\mathrm{Id}(\mu \mathrm{A})(\mathrm{Vds}=\mathrm{Vgs}=-5 \mathrm{~V})$ & 3.01 & 2.60 & 2.14 & 1.19 \\
Hooge's parameter $\alpha$ & 0.035 & 0.021 & 0.086 & 0.440 \\
tox $(\mathrm{nm})$ & 39.2 & 40.1 & 39.7 & 38.1 \\
Cox $\left(\mu \mathrm{F} / \mathrm{cm}^{2}\right)$ & 0.228 & 0.230 & 0.218 & 0.228 \\
$\mathrm{k}$ value & 10.1 & 10.4 & 9.8 & 9.8 \\
\hline \hline
\end{tabular}

$1 / f$ noise reveals the current fluctuation in the conduction channel and is closely related to the transport of carriers. Since organic devices may focus on low-frequency applications, $1 / f$ noise is of significant concern due to the fact that noise power becomes larger in the lower frequency regime. Fig. 3 shows the comparison of the $1 / f$ noise spectrum for the OTFTs. The $\mathrm{NH}_{3}$-annealed OTFT has the least $1 / f$ noise among the samples. According to the Hooge's expression for the $1 / f$ noise of OTFTs [13], the Hooge's parameter $\alpha$ can be calculated as

$$
\alpha=\frac{f^{\gamma} S_{\mathrm{id}}(f) L^{2}}{e \mu V_{\mathrm{ds}} I_{d}}
$$

where $S_{\text {id }}(f)$ is the power spectral density and $f$ is the measurement frequency. The $\mathrm{NH}_{3}$-annealed OTFT has a Hooge parameter of 0.021 , which is the smallest among the devices, hence the lowest noise level.

The device parameters of the OTFTs and capacitors are summarized in Table I. The higher carrier mobility, smaller SS, and lower $1 / f$ noise level of the $\mathrm{NH}_{3}$-annealed OTFT can be attributed to the improved interfacial characteristics between the organic film and the gate dielectric obtained by annealing the latter in $\mathrm{NH}_{3}$. The reason should be that the hydrogen atoms decomposed from the $\mathrm{NH}_{3}$ gas could passivate the dangling bonds at the surface of the HfLaO film and thus reduce the interface traps. This can be supported by Fig. 4, which shows the $C-V$ characteristics (measured at $1-\mathrm{MHz}$ frequency) of the $\mathrm{Au}$-pentacene-HfLaO-Si unit with the dielectric annealed in $\mathrm{N}_{2}, \mathrm{NH}_{3}, \mathrm{O}_{2}$, or NO. In Fig. 4, the $\mathrm{NH}_{3}$-annealed unit displays the smallest hysteresis, while the NO-annealed structure has the largest hysteresis. The morphology of the pentacene film is shown in Fig. 5. From Figs. 4 and 5, the NO annealing is undesirable because it gives the largest hysteresis and smallest grains. 

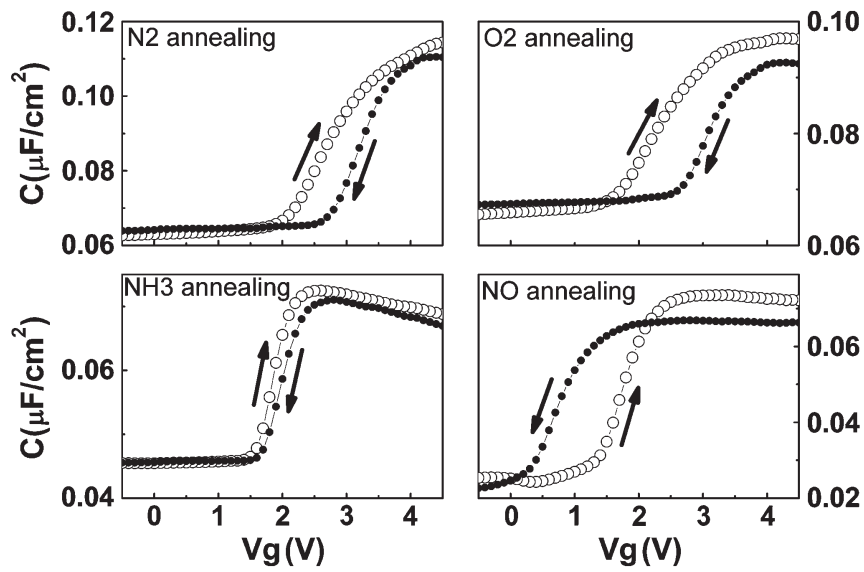

Fig. 4. $C-V$ characteristics of the Au-pentacene-HfLaO-Si units.
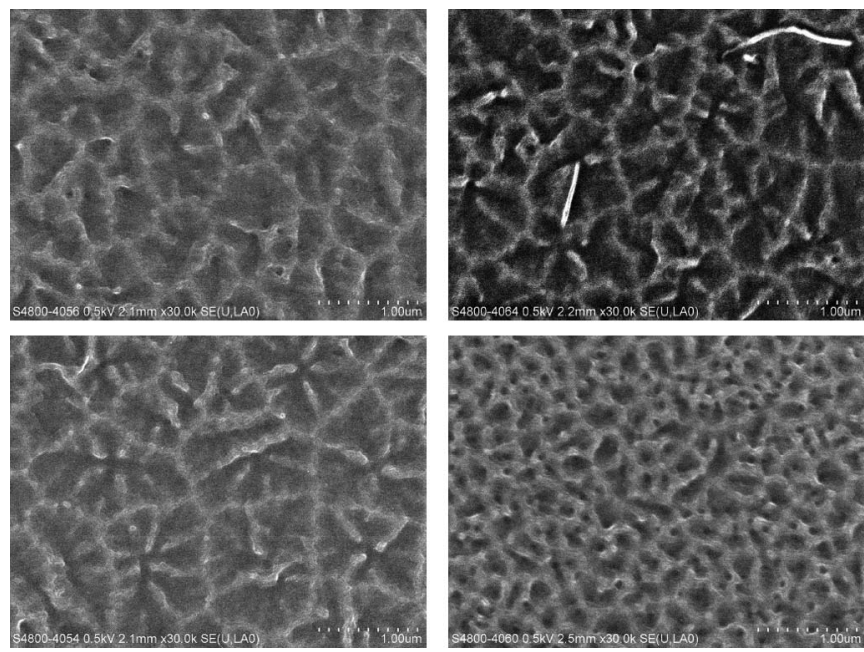

Fig. 5. SEM images of the pentacene film grown on the dielectric. (Northwest) $\mathrm{N}_{2}$ annealing. (Northeast) $\mathrm{O}_{2}$ annealing. (Southwest) $\mathrm{NH}_{3}$ annealing. (Southeast) NO annealing.

In [11], pentacene OTFT with a more advanced structure was based on a $\mathrm{HfLaO}$ gate dielectric annealed in $\mathrm{O}_{2}$, and its carrier mobility and SS were reported to be $0.71 \mathrm{~cm}^{2} / \mathrm{V} \cdot \mathrm{s}$ and $0.078 \mathrm{~V} / \mathrm{dec}$, respectively. Therefore, this study could provide a method to further improve the performance of the OTFT by annealing the HfLaO gate dielectric in $\mathrm{NH}_{3}$. The OTFTs in this study display a lower on/off current ratio than that reported in [11]. One reason is that a patterned gate structure was used in [11]. Another possible reason is that the HfLaO film prepared by electron beam evaporation of compound HfLaO source [11] might achieve smaller dielectric leakage than our film made by sputtering of a metal target. Nevertheless, since our work is mainly related to the postdeposition treatment of the dielectric film, the device improvements mentioned previously should also be applicable to dielectric films fabricated by other techniques such as electron beam evaporation or atomic layer deposition.

\section{REFERENCES}

[1] C. D. Dimitrakopoulos, S. Purushothaman, J. Kymissis, A. Callegari, and J. M. Shaw, "Low-voltage organic transistors on plastic comprising highdielectric constant gate insulators," Science, vol. 283, no. 5403, pp. 822824, Feb. 1999.

[2] B. Crone, A. Dodabalapur, Y.-Y. Lin, R. W. Filas, Z. Bao, A. LaDuca, R. Sarpeshkar, H. E. Katz, and W. Li, "Large-scale complementary integrated circuits based on organic transistors," Nature, vol. 403, no. 6769 , pp. 521-523, Feb. 2000.

[3] C. D. Sheraw, L. Zhou, J. R. Huang, D. J. Gundlach, T. N. Jackson, M. G. Kane, I. G. Hill, M. S. Hammond, J. Campi, B. K. Greening, J. Francl, and J. West, "Organic thin-film transistor-driven polymerdispersed liquid crystal displays on flexible polymeric substrates," Appl. Phys. Lett., vol. 80, no. 6, pp. 1088-1090, Feb. 2002.

[4] W. Fix, A. Ullmann, J. Ficker, and W. Clemens, "Fast polymer integrated circuits," Appl. Phys. Lett., vol. 81, no. 9, pp. 1735-1737, Aug. 2002.

[5] S. F. Nelson, Y.-Y. Lin, D. J. Gundlach, and T. N. Jackson, "Temperatureindependent transport in high-mobility pentacene transistors," Appl. Phys. Lett., vol. 72, no. 15, pp. 1854-1856, Apr. 1998.

[6] A. Facchetti, M.-H. Yoon, and T. J. Marks, "Gate dielectrics for organic filed-effect transistors: New opportunities for organic electronics," $A d v$. Mater, vol. 17, no. 14, pp. 1705-1725, Jul. 2005.

[7] C. Bartic, H. Jansen, A. Campitelli, and S. Borghs, " $\mathrm{Ta}_{2} \mathrm{O}_{5}$ as gate dielectric material for low-voltage organic thin-film transistors," Org. Electron., vol. 3, no. 2, pp. 65-72, Jun. 2002.

[8] G. Wang, D. Moses, A. J. Heeger, H.-M. Zhang, M. Narasimhan, and R. E. Demaray, "Poly(3-hexylthiophene) field-effect transistors with high dielectric constant gate insulator," J. Appl. Phys., vol. 95, no. 1, pp. 316322, Jan. 2004.

[9] X. P. Wang, C. Shen, M.-F. Li, H. Y. Yu, Y. Sun, Y. P. Feng, A. Lim, H. W. Sik, A. Chin, Y. C. Yeo, P. Lo, and D. L. Kwong, "Dual metal gates with band-edge work functions on novel HfLaO high- $\kappa$ gate dielectric," in VLSI Symp. Tech. Dig., 2006, pp. 12-13.

[10] X. P. Wang, H. Y. Yu, M.-F. Li, C. X. Zhu, S. Biesemans, A. Chin, Y. Y. Sun, Y. P. Feng, A. Lim, Y.-C. Yeo, W. Y. Loh, G. Q. Lo, and D.-L. Kwong, "Wide $V_{\mathrm{fb}}$ and $V_{\mathrm{th}}$ tunability for metal-gated MOS devices with HfLaO gate dielectrics," IEEE Electron Device Lett., vol. 28, no. 4, pp. 258-260, Apr. 2007.

[11] M. F. Chang, P. T. Lee, and S. P. McAlister, "Low subthreshold swing $\mathrm{HfLaO}$ /pentacene organic thin-film transistors," IEEE Electron Device Lett., vol. 29, no. 3, pp. 215-217, Mar. 2008.

[12] F.-C. Chen, C.-W. Chu, J. He, Y. Yang, and J.-L. Lin, "Organic thinfilm transistors with nanocomposite dielectric gate insulator," Appl. Phys. Lett., vol. 85, no. 15 , pp. 3295-3297, Oct. 2004.

[13] M. J. Deen, O. Marinov, J. Yu, S. Holdcroft, and W. Woods, "Lowfrequency noise in polymer transistors," IEEE Trans. Electron Devices, vol. 48, no. 8, pp. 1688-1694, Aug. 2001. 